\title{
High-Brightness Photocathode Electron Sources
}

\author{
Christian Travier \\ Laboratoire de l'Accélérateur Linéaire \\ IN2P3-CNRS et Université de Paris-Sud \\ Bâtiment 200 \\ F-91405 Orsay
}

\begin{abstract}
Most present and future electron accelerators require bright sources. Invented less than ten years ago, the photo-injector the principle of which is briefly recalled, has already demonstrated that it can provide very bright beams. In this paper, the most advanced photo-injector projects are reviewed, their specific features are outlined, and their major issues are examined. The state-of-the-art in photocathode and laser technologies is presented. Beam dynamics issues are also considered since they are essential in the production of bright beams. Finally, the question of the maturity of photo-injector technology is addressed.
\end{abstract}

\section{INTRODUCTION}

Numerous applications of electron linacs require high-brightness sources. These include high-energy linear colliders ${ }^{1}$, short wavelength free electron lasers ${ }^{2}$, wakefield accelerator experiments ${ }^{3}$, new accelerator scheme test facilities ${ }^{4}$, drive beam for two-beam accelerators $(\mathrm{TBA})^{5}$, coherent radiation sources $^{6}$, radiochemistry $^{7}, \ldots$

The brightness being proportional to the peak current divided by the square of the normalized emittance, bright electron sources require intense beams (high charge and short pulse) and small emittances. Table 1 indicates qualitatively the needs for the different applications, since they do not all require the same kind of beams. Figure 1 shows the normalized brightness needed by linear colliders and FEL applications. It also displays the present state-of-the-art of conventional injectors (DC gun + bunchers), thermionic RF guns and photo-injectors. This plot tends to show that a photo-injector allows on average two (respectively one) orders of magnitude improvement in brightness when compared to a conventional injector (respectively thermionic RF gun).

After a brief description of photo-injector principle and a short historical overview, this paper focuses on the nine most advanced projects. General remarks concerning current trends of R\&D in the field of photocathodes, lasers, and beam dynamics in a photo-injector are then discussed. 


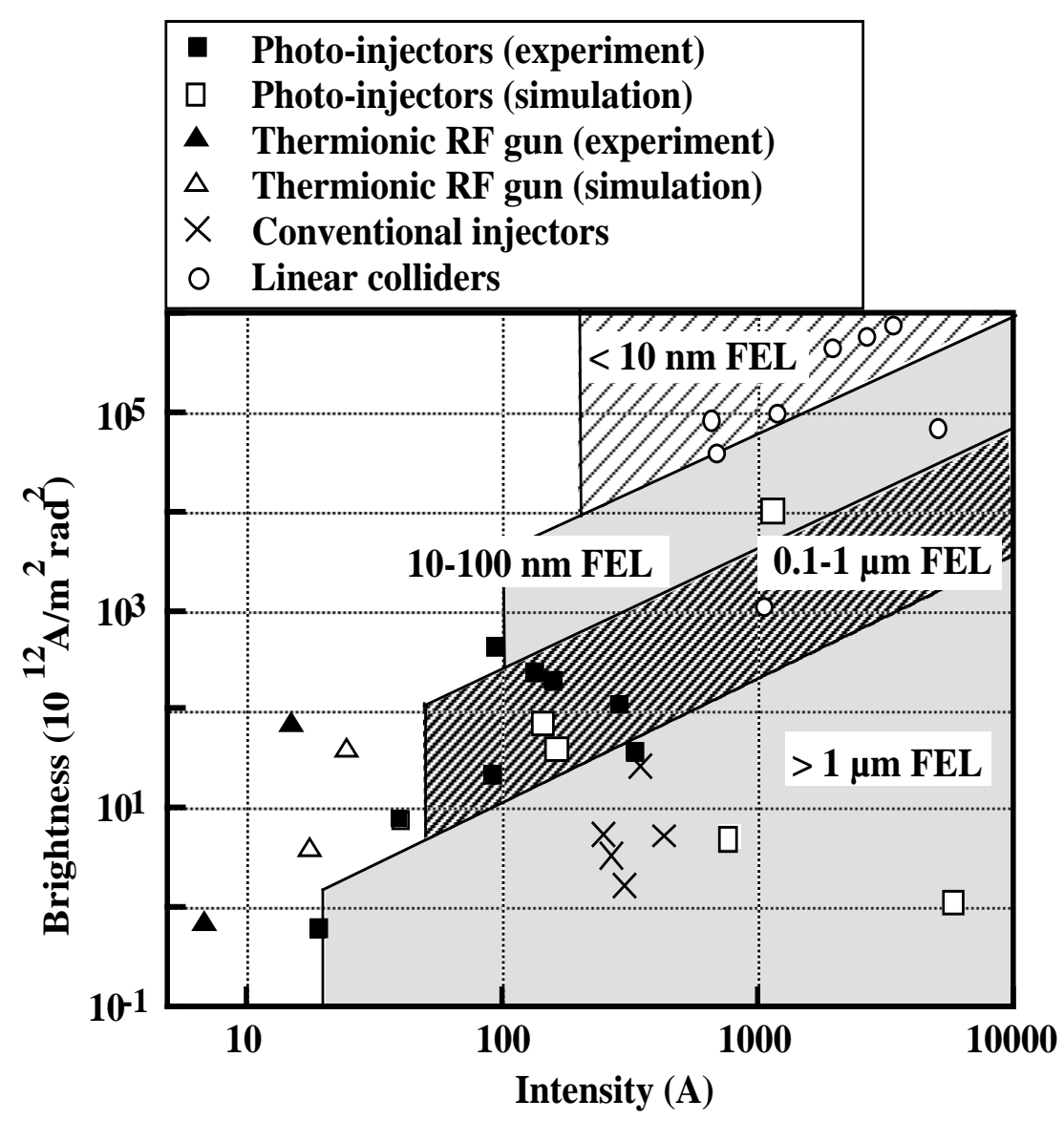

Figure 1: Brightness required for FEL and linear colliders

Table 1: Beam requirements for future applications

\begin{tabular}{|c|c|c|c|c|}
\hline & $\begin{array}{l}\text { High } \\
\text { charge }\end{array}$ & $\begin{array}{l}\text { Low } \\
\text { emittance }\end{array}$ & $\begin{array}{l}\text { Short } \\
\text { pulse }\end{array}$ & Other \\
\hline Linear colliders & $*$ & $* * *$ & **** & polarized, asymmetric \\
\hline FEL & $* *$ & $* *$ & * & $\begin{array}{l}\text { high average power, } \\
\text { very good phase and } \\
\text { amplitude stability }\end{array}$ \\
\hline TBA, RF sources & $* * *$ & & ** & \\
\hline Wakefield accelerators & $* * *$ & & ** & \\
\hline New acceleration schemes & & ** & * & \\
\hline Coherent radiation sources & & $*$ & $* * *$ & high average power \\
\hline Radiochemistry & $* * *$ & & $* *$ & \\
\hline
\end{tabular}




\section{PHOTO-INJECTOR PRINCIPLE}

To increase the brightness of an electron source, it is necessary to increase its peak current while keeping a very small transverse emittance. This leads to the use of high electric fields to reduce the influence of space charge forces. Since DC voltages in a gun are limited to a few hundred kilovolts, it is more appropriate to use RF fields to extract high peak current from a cathode. Following this idea, G. Westenskow and J. Madey designed and operated in 1985, the first microwave gun consisting of a thermionic cathode located in an S-band RF cavity ${ }^{19}$. Meanwhile, the necessity of high peak current implies the use of short pulses. The electronic switching of the grid in a conventional DC gun does not allow one to produce pulses shorter than a few hundred picoseconds. To obtain shorter pulses, it is natural to think about optical switching. A short laser pulse illuminating a photocathode provides an almost ideal way to produce such short pulses. It was first experimented with at Los Alamos by Fraser and Sheffield ${ }^{8}$. The combination of acceleration in a high RF field and generation of electrons by short laser pulses hitting a photocathode make a quasi perfect bright injector. Today, lasers are able to produce very short pulses (down to less then 1 $\mathrm{ps}$ ), photocathodes can deliver high current densities (several thousands of $\mathrm{kA} / \mathrm{cm}^{2}$ ) and RF cavities can sustain electric fields as high as $100 \mathrm{MV} / \mathrm{m}$, so that RF photo-injectors can reach very high brightness.

This potentiality of photo-injectors to produce bright beams has therefore boosted their development. Since their invention 10 years ago, the number of photo-injectors has rapidly increased and currently exceeds 30 . Table 2 gives a summary of the main breakthroughs occured during the last ten years in this quickly progressing field of RF photo-injectors.

Table 2: Main breakthroughs in photo-injector history

\begin{tabular}{llc}
\hline \hline Date & Event & Reference \\
\hline 1985 & First photo-injector at Los Alamos & 8 \\
1988 & First FEL driven by photo-injector at Stanford & 9 \\
1988 & Emittance compensation theory by B. Carlsten & 10 \\
1989 & First S-band photo-injector at Brookhaven & 11 \\
1989 & Analytic theory of photo-injector beam dynamics by K.J. & 12 \\
& Kim & 13 \\
1990 & First 144 MHz photo-injector at CEA & 14 \\
1992 & First 433 MHz high duty cycle photo-injector at BOEING & 15 \\
1992 & First photocathode in a superconducting RF cavity at & 16 \\
& Wuppertal & 17 \\
1993 & UV FEL driven by photo-injector at Los Alamos & 18 \\
1993 & First sub-picosecond laser driven photo-injector at LAL & \\
1994 & New analytic theory of photo-injector beam dynamics by L. & \\
& Serafini & \\
\hline \hline
\end{tabular}

For the sake of completness, it should be noted here, that it is also possible to use a photocathode in a DC gun, thus making a DC photo-injector. Such a device could in principle deliver short pulses. However, due to the moderate accelerating gradient, only 
very low charge can be extracted in short pulse. Practically, DC photo-injectors are used to produce intense pulses of a few hundred picoseconds to a few nanoseconds, and therefore still necessitate RF bunchers. The DC photo-injector has been extensively studied at SLAC, where a $120 \mathrm{kV}$ gun using a GaAs photocathode is delivering daily polarized electrons to the $\mathrm{SLC}^{20}$.

\section{REVIEW OF ADVANCED PHOTO-INJECTORS}

The most advanced photo-injector projects are reviewed below. The main features of each project are discussed and table 3 summarizes their parameters. Except for ANL and MIT, these results correspond to experimental data and represent consistent sets of typical parameters. One should therefore be very careful while comparing these data since some of them (eg. emittance) are rather difficult to measure.

\section{Los Alamos National Laboratory}

As already mentioned, the first photo-injector was designed and built at Los Alamos by J. Fraser and R. Sheffield ${ }^{8}$. After this first prototype, LANL has built APEX ${ }^{21}$ and AFEL ${ }^{22}$ photo-injectors. These devices are sophisticated guns made of several RF cells and using the emittance compensation scheme devised by B. Carlsten ${ }^{10}$. The APEX gun brightness was so high that it allowed the first UV FEL lasing on a linac ${ }^{16}$. The AFEL gun is made of 11 cells (see figure 2) and produces a very bright electron beam used to drive a very

compact FEL. Present studies include a detailed understanding of emittance measurements for these very bright beams.

\section{Brookhaven National Laboratory}

The BNL gun design ${ }^{23}$ shown in figure 3 is the most popular one, since it has already been reproduced 10 times. The careful design of the cavity shape is intended to completely suppress higher spatial harmonics of the field, thus minimizing the non-linear emittance. The high gradient operation (up to $100 \mathrm{MV} / \mathrm{m}$ ) allows one to produce the very small emittance beams needed by the FEL and advanced accelerator physics experiments done at the Brookhaven ATF facility.

The most oustanding recent result is the convenient use of a magnesium cathode, that proved both to have a relatively good quantum efficiency $\left(5 \times 10^{-4}\right)$ and be very robust (lifetime over 5000 hours) $^{24}$.

This one and a half cell gun is now being replaced by a three and a half cell gun jointly designed and fabricated by BNL and Grumman ${ }^{25}$ and that can work at very high duty cycle (1\%). A new laser system is also being assembled (see the parameters in table 5). Together with SLAC, UCLA, NRL, and LANL, BNL is now designing an inexpensive gun that would allow smaller laboratories or universities and smaller groups inside big laboratories to afford such a bright gun for any type of application. 
Table 3: Parameters for the main photo-injectors

\begin{tabular}{|c|c|c|c|c|c|c|c|c|c|}
\hline Parameter & CEA & LANL & ANL & $\mathrm{BNL}$ & CERN & KEK & UCLA & LAL & MIT \\
\hline Purpose & FEL & FEL & $\begin{array}{l}\text { Wakefield } \\
\text { accelerator }\end{array}$ & $\begin{array}{l}\text { Advanced } \\
\text { accelerator }\end{array}$ & $\begin{array}{l}\text { Linear } \\
\text { collider }\end{array}$ & $\begin{array}{l}\text { Linear } \\
\text { collider }\end{array}$ & FEL & $\begin{array}{l}\text { Linear } \\
\text { collider }\end{array}$ & $\begin{array}{l}\text { High } \\
\text { gradient }\end{array}$ \\
\hline First operation & 1990 & 1992 & - & 1989 & 1990 & $?$ & $?$ & 1993 & - \\
\hline Running time (h) & 3200 & 1000 & 0 & 5000 & 1800 & $?$ & $?$ & 100 & 0 \\
\hline Number of cavities & 1 & 11 & 1 & 1.5 & 1.5 & 1 & 1.5 & 2 & 1.5 \\
\hline Frequency (MHz) & 144 & 1300 & 1300 & 2856 & 2998 & 2856 & 2856 & 2998 & 17136 \\
\hline Macropulse $(\mu \mathrm{s})$ & $20-160$ & 20 & 8 & 3 & 2.5 & 2 & 4 & 3 & $0.05-1$ \\
\hline Repetition rate $(\mathrm{Hz})$ & $1-10$ & 20 & 30 & $1.5-6$ & 10 & 5 & 10 & 12.5 & $1-4$ \\
\hline Vacuum RF off (nTorr) & 1 & 0.2 & 100 & 10 & 0.1 & $?$ & $?$ & 10 & 2 \\
\hline Vacuum RF on (nTorr) & 2.5 & 1 & $?$ & 50 & 1 & $?$ & $?$ & 50 & 10 \\
\hline Cathode field (MV/m) & 28 & 20 & 90 & 70 & 100 & 40 & 83 & 50 & 250 \\
\hline Cathode & $\mathrm{Cs}_{3} \mathrm{~K}_{2} \mathrm{Sb}$ & $\mathrm{Cs}_{2} \mathrm{Te}$ & $\mathrm{Y}$ & $\mathrm{Cu}$ & $\mathrm{Cs}_{2} \mathrm{Te}$ & $\mathrm{CsSb}$ & $\mathrm{Cu}$ & $\mathrm{Cu}$ & $\mathrm{Cu}$ \\
\hline Quantum efficiency (\%) & 3 & 5 & 0.05 & 0.001 & 2 & $?$ & $?$ & $5 \times 10^{-4}$ & 0.001 \\
\hline Lifetime & $1 \mathrm{~h}$ & months & $?$ & $\infty$ & $70 \mathrm{~h}$ & $?$ & $\infty$ & $\infty$ & $?$ \\
\hline Laser & YAG & YLF & $\mathrm{Kr}-\mathrm{F}$ & YAG & YLF & YAG & YAG & Ti:sa & Ti:sa \\
\hline Wavelength (nm) & 532 & 263 & 248 & 266 & 262 & 532 & 266 & 260 & 260 \\
\hline Pulse length FWHM (ps) & $20-50$ & 6 & 3 & 15 & 8 & 10 & 4 & 0.5 & 2 \\
\hline Energy single pulse $(\mu \mathrm{J})$ & 20 & 50 & 12000 & 300 & 1 & 100 & 300 & 250 & 200 \\
\hline Spot size FWHM (mm) & $2-7$ & 4 & 20 & $0.1-1$ & 5 & $?$ & 0.6 & 4 & 1 \\
\hline Energy $(\mathrm{MeV})$ & 2 & 16 & 1.7 & 3 & 4 & 0.9 & 3.5 & 2.2 & 2.8 \\
\hline Charge $(\mathrm{nC})$ & $0.5-5$ & 3 & 100 & 0.5 & 4 & 3.2 & 0.5 & 0.11 & $0.1-1$ \\
\hline Pulse length FWHM (ps) & $20-50$ & 20 & 14 & 11 & 13 & $?$ & 5 & $?$ & 1.5 \\
\hline Jitter $(\mathrm{ps})$ & 3 & $<1$ & $<10$ & $<1$ & $<1$ & $?$ & $?$ & $?$ & 2 \\
\hline $\begin{array}{l}\text { Normalized rms emittance ( } \pi \\
\mathrm{mm} \text { mrad) }\end{array}$ & 4@1nC & 5 & 130 & 4 & $?$ & $?$ & 10 & $?$ & $3 @ 1 n C$ \\
\hline
\end{tabular}




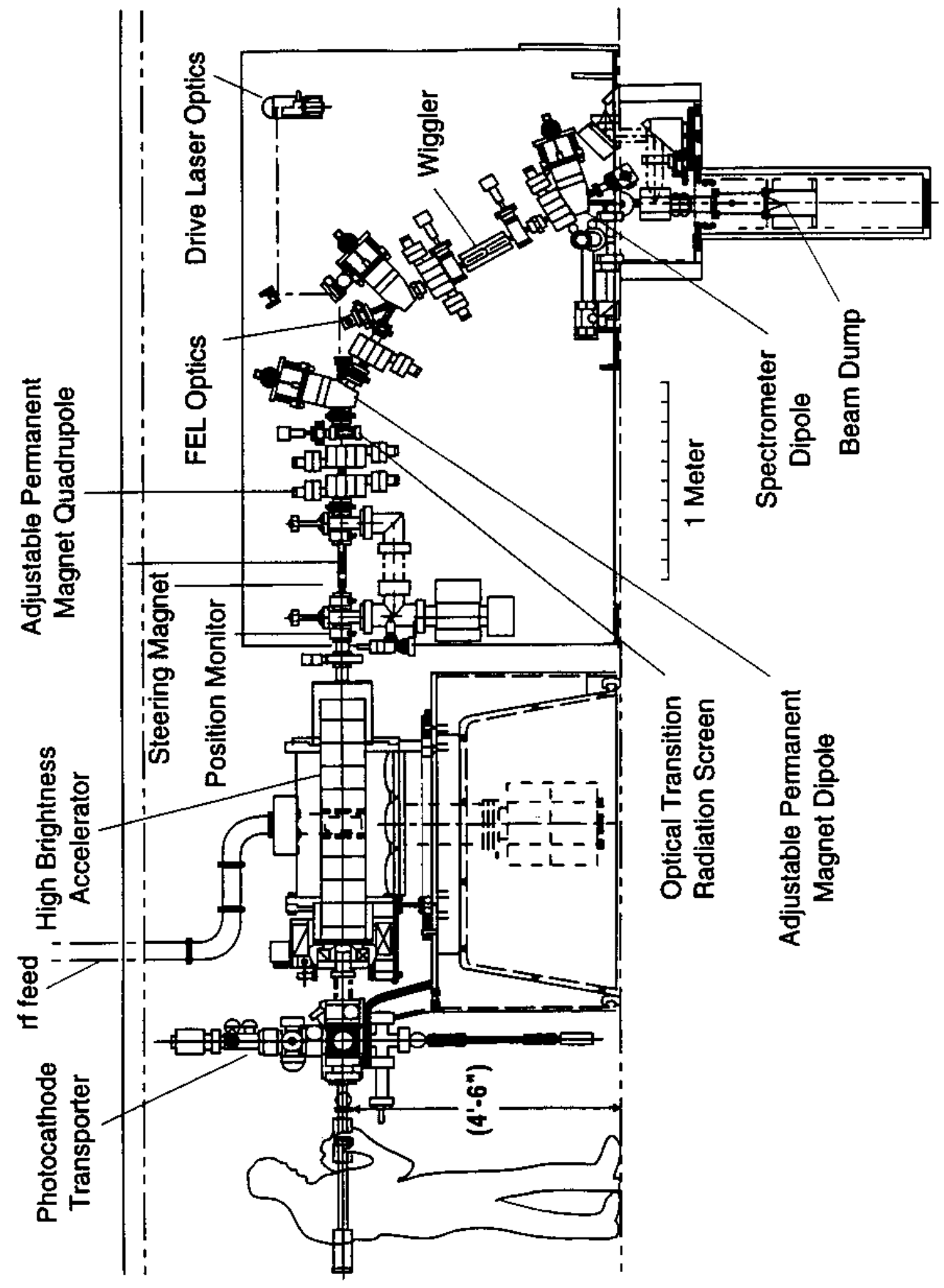

Figure 2: Schematic of the AFEL experiment 


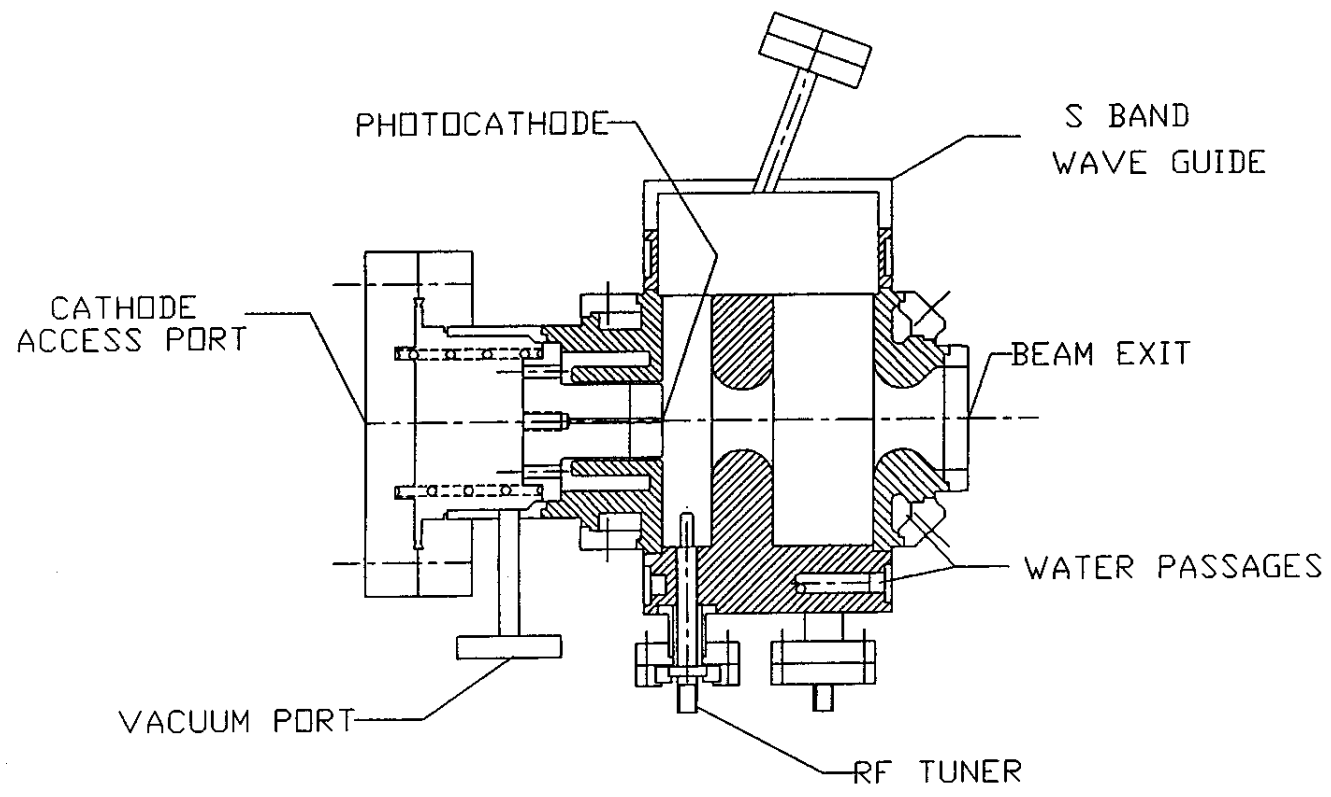

Figure 3: BNL one and a half cell gun

\section{CEA at Bruyères-le-Châtel}

At CEA, the photo-injector is made of one $144 \mathrm{MHz}$ cavity and is used as the electron source for the infra-red high power $\mathrm{FEL}^{13}$. Recent emphasis has been put on improving the stability and reliability of the system. An amplitude feedback system is being developed to improve the laser stability.

\section{CERN}

The CTF (CLIC Test Facility) was built at CERN to test some components of the CERN Linear Collider project ${ }^{26}$ based on the concept of the two-beam accelerator. In order to generate the RF power at $30 \mathrm{GHz}$, necessary to obtain the high accelerating gradient needed for the $30 \mathrm{GHz}$ CLIC accelerating section, a train of short intense electron bunches is accelerated, and then produces RF power through electromagnetic interaction with a socalled tranfer structure. This train of intense short electron pulses is generated by a BNL type RF gun, using a $\mathrm{Cs}_{2}$ Te photocathode. Recently a train of 24 pulses, 14 ps long and 6.2 $\mathrm{nC}$ each (at gun exit) led to the production of $40 \mathrm{MW}$ of $30 \mathrm{GHz} \mathrm{RF}$ power ${ }^{27,28}$. Figure 4 shows a schematic of the experiment.

When running with a single pulse, a charge as high as $26 \mathrm{nC}$ was extracted from this photoinjector. The $\mathrm{Cs}_{2} \mathrm{Te}$ photocathode presents a good combination of a very high quantum efficiency $(2-5 \%)$ and a long lifetime (several months). 


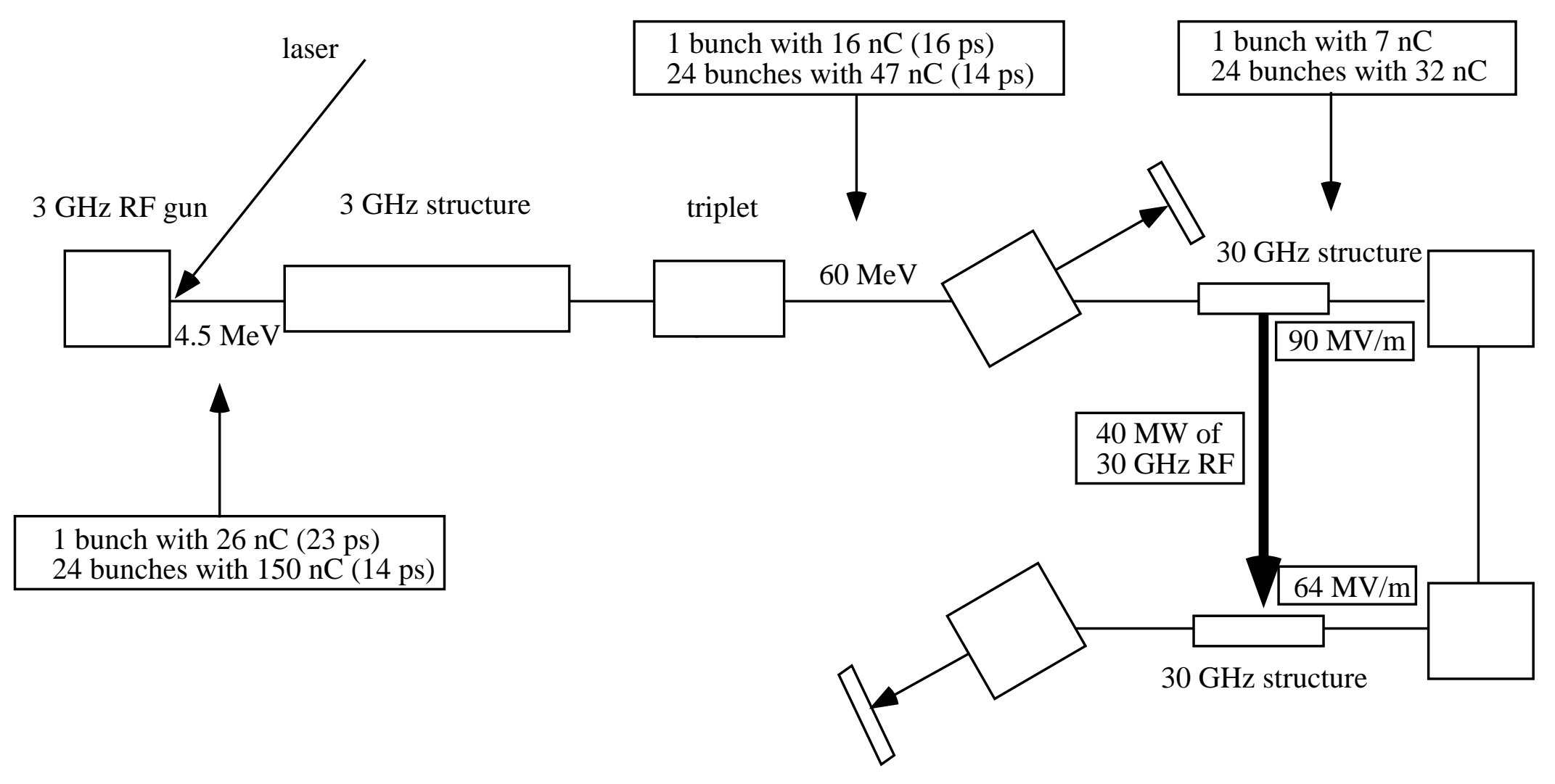

Figure 4: Schematic layout of CLIC Test Facility 


\section{KEK}

A 1 cell S-band RF gun was developped at KEK in view of JLC linear collider ${ }^{29}$. This gun is the only one working with relatively high accelerating gradient ( $>40 \mathrm{MV} / \mathrm{m}$ ) and using an alcaline cathode ( $\mathrm{CsSb}$ ). A special emphasis has therefore been put on the vacuum system design with the use of NEG pumping. Since linear colliders need polarized sources, KEK is working on the subject of polarized photocathodes ${ }^{30}$ and polarized RF gun in collaboration with SLAC ${ }^{38}$.

\section{University of California at Los Angeles}

The UCLA RF gun is a modified BNL gun. It allows 70 degree laser illumination of the cathode which produces an enhancement of the quantum efficiency when compared to normal incidence. Extensive measurements and comparison to simulation were made and proved to be satisfactory ${ }^{31}$.

\section{Laboratoire de l'Accélérateur Linéaire Orsay}

A two decoupled cell S-band RF gun was recently put into operation at $\mathrm{LAL}^{17}$. The cathode is illuminated by a Ti:sapphire laser shown in figure 5, that produces 0.5 ps pulses $^{32}$. This project is the first sub-picosecond laser driven photo-injector. Though these preliminary results were obtained with a copper cathode, a dispenser cathode $(\mathrm{WCaOBaO})$ used as a photoemitter will soon be installed in the gun. This cathode has a measured quantum efficiency of $5 \times 10^{-4}$ with UV light and at room temperature ${ }^{33}$.

\section{Argonne National Laboratory}

In order to do wakefield accelerator experiments, it is necessary to generate very intense and short electron bunches ${ }^{3}$. At ANL, a photo-injector designed to produce $100 \mathrm{nC}$ pulses is being commissioned. To maintain a pulse as short as possible in spite of the enormous space charge forces, a concave shape of the laser wavefront is created ${ }^{34}$. To probe the wakefield excited by this intense pulse, a second pulse is generated by a 7 cell photo-injector ${ }^{35}$. An alternative design for this witness beam gun is a dielectric loaded cavity that allows one to produce a perfectly linear accelerating field ${ }^{35}$.

\section{Massachussets Institute of Technology}

The MIT photo-injector is a one and a half cell BNL type gun scaled at $17 \mathrm{GHz}^{36}$. The use of such a high frequency makes very high accelerating gradient possible. $250 \mathrm{MV} / \mathrm{m}$ is envisaged for this gun presently under commissioning.

\section{PHOTOCATHODES}

A good photocathode for photo-injector operation should ideally have a high quantum efficiency $(>1 \%)$ at infra-red or visible wavelength, have a long lifetime ( $>$ several 


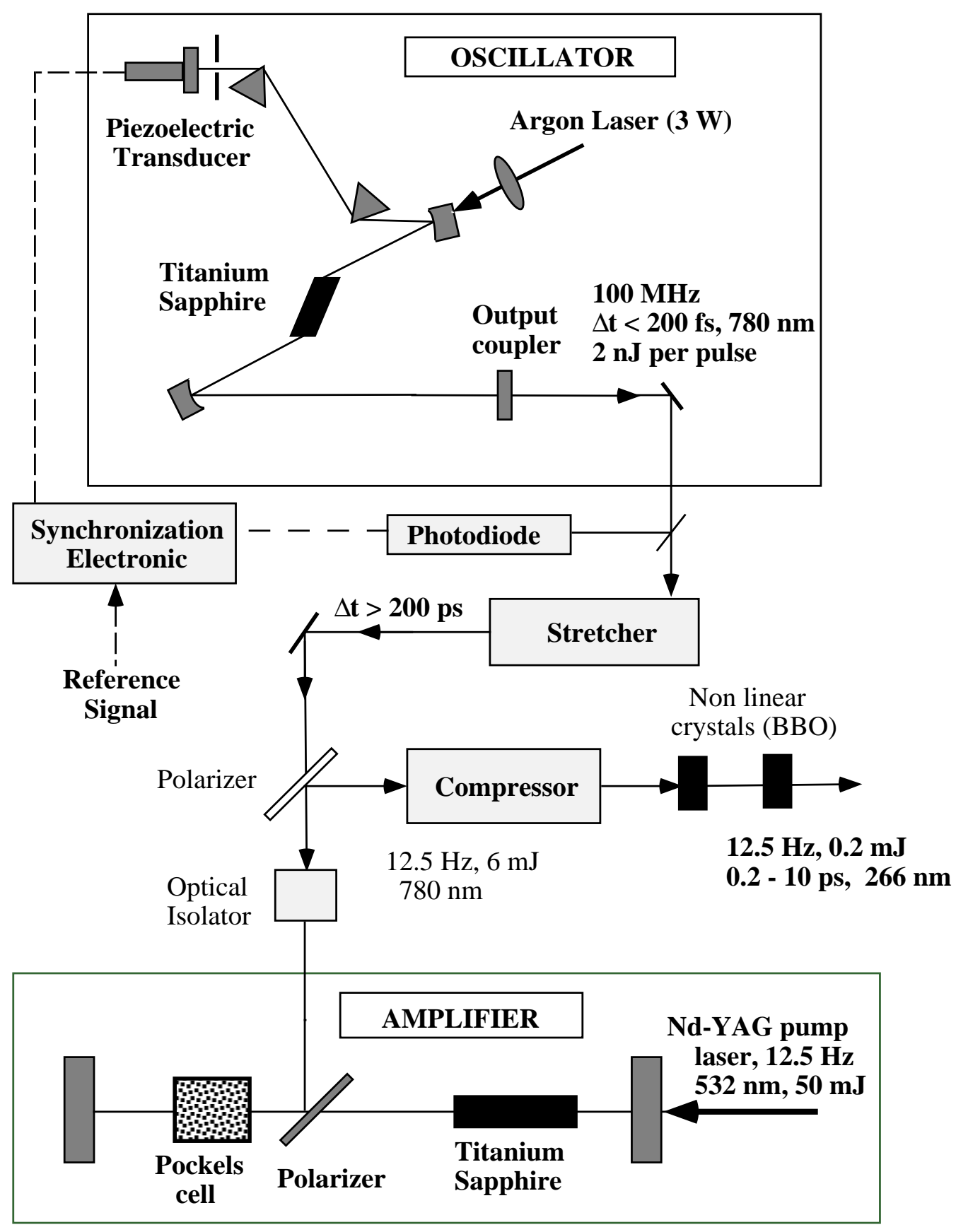

Figure 5: LAL Orsay Ti:sapphire femtosecond laser 
months) under moderate vacuum conditions, and be easy to prepare and install in the gun cavity. Such a perfect cathode does not exist yet, but progress was made recently, especially with $\mathrm{Cs}_{2} \mathrm{Te}$ and $\mathrm{Mg}$ cathodes. Table 4 shows the most commonly used photocathodes and a summary of their main advantages and drawbacks.

Table 4: Main photocathodes used in photo-injectors

\begin{tabular}{|c|c|c|}
\hline Cathode & Advantage & Drawback \\
\hline $\mathrm{Cs}_{3} \mathrm{Sb}, \mathrm{CsK}_{2} \mathrm{Sb}$ & $\begin{array}{l}\text { high quantum efficiency } \\
0.5 \mu \mathrm{m} \text { laser }\end{array}$ & $\begin{array}{l}\text { difficult to prepare } \\
\text { short lifetime } \\
\text { do not sustain very high fields } \\
\text { expensive preparation chamber } \\
\text { and transfer system } \\
\text { need good vacuum }\end{array}$ \\
\hline $\mathrm{Cs}_{2} \mathrm{Te}$ & $\begin{array}{l}\text { high quantum efficiency } \\
\text { long lifetime } \\
\text { sustains high fields }\end{array}$ & $\begin{array}{l}\text { needs UV laser } \\
\text { expensive preparation chamber } \\
\text { and transfer system } \\
\text { response to train? }\end{array}$ \\
\hline $\mathrm{Cu}, \mathrm{Y}, \mathrm{Mg}$ & $\begin{array}{l}\text { no preparation chamber } \\
\text { long lifetime } \\
\text { fast response } \\
\text { sustain very high fields } \\
\text { sustain bad vacuum }\end{array}$ & $\begin{array}{l}\text { low quantum efficiency (except } \\
\mathrm{Mg} \text { ) } \\
\text { need UV laser }\end{array}$ \\
\hline $\mathrm{LaB}_{6}, \mathrm{WCaOBaO}$ & $\begin{array}{l}\text { no preparation chamber } \\
\text { long lifetime }\end{array}$ & $\begin{array}{l}\text { low quantum efficiency } \\
\text { need UV laser } \\
\text { need to be heated prior to } \\
\text { operation }\end{array}$ \\
\hline
\end{tabular}

Specific issues are to be considered for two particular applications: polarized sources and superconducting photo-injectors.

- The only photocathode presently available to generate polarized electron is GaAs. Bulk GaAs gives polarization of $40 \%$, where strained layers of GaAs can lead to polarization as high as $90 \%$. This type of cathode under extensive study ${ }^{37}$, has never been used in an RF gun so far ${ }^{38}$. Many limitations might appear in doing so in order to produce short intense polarized electron beams. Besides technological problems such as sensitivity of the cathode to vacuum conditions or high gradient, there are more fundamental problems such as the charge limit effect discovered and explained at $\mathrm{SLAC}^{20}$, and the time response to picosecond pulses ${ }^{39}$. These problems are now being investigated since polarized electron sources is an important challenge of any linear collider project.

- Using superconducting cavities for an RF gun is interesting in some cases, as for example very high duty cycle machines. In this case, one has to find a photocathode that can work in a superconducting environment, i.e. introducing the following specific 
requirements: work function below that of niobium $(4.6 \mathrm{eV})$ to avoid photoemission from the surface of the cavity, and very low e.m. losses at cryogenic temperature in order to preserve the very high quality factor of the cavity. The first experimental work done at Wuppertal has shown that a $\mathrm{CsK}_{2} \mathrm{Sb}$ cathode has better quantum efficiency at cryogenic temperature than at room temperature. It also showed that if the cathode is too thick, it is not superconducting and thus leads to very high ohmic losses ${ }^{15}$. Following this work, studies are now made at Milan to produce very thin $(<30 \mathrm{~nm})$ cathodes, that can become superconducting by proximity effects ${ }^{40}$.

The choice of the photocathode to be used depends on the type of applications. It depends of course on the charge required from a single pulse, but also on the pulse format via the existence or not of a suitable laser. The typical cases are the following:

- if a single pulse of charge below $5 \mathrm{nC}$, is required, a $\mathrm{Mg}$ photocathode is probably the best choice.

- if train a pulses of charge less than a few $\mathrm{nC}$ are required, then one should probably go to $\mathrm{Cs}_{2} \mathrm{Te}$.

- for very high repetition rate or very high duty cycle and high charge, it is probably difficult to avoid $\mathrm{CsK}_{2} \mathrm{Sb}$.

\section{LASERS}

One of the key components of a photo-injector system is the laser. Amplitude, phase and position stability of the electron beam depend almost completely on the laser performances. A laser is typically made of an oscillator that generates a continuous train of pulses of low energy (few $\mathrm{nJ}$ ). This oscillator is synchronized via an appropriate electronic system to a sub-harmonic of the RF frequency (typically $100 \mathrm{MHz}$ ). One single pulse or a train of a few hundred pulses is then selected through a Pockells cell and amplified. There exist several types of amplifiers (single pass, multiple pass, regenerative, ...) and according to the energy desired, it might be necesssary to have several amplifier stages. When the oscillator pulse is very short, one has to extend the pulse temporally before amplification to avoid damage of the amplifier cavity components. The pulse is then compressed back to its original duration. This technique is called chirped pulse amplification. The oscillators used so far for photo-injector applications produce infra-red light. To obtain usable light for the photo-cathode, it is therefore necessary to generate higher harmonics. This is done by using non-linear crystals, with a typical efficiency of $10-15 \%$ from the fundamental to the third or fourth harmonic.

$\mathrm{Nd}$ :YAG and Nd:YLF (eg. reference 41) are the most commonly used systems in existing photo-injectors. They typically produce pulses of 6-15 ps, with up to $300 \mu \mathrm{J}$ of energy in a single pulse. More recently the advent of Ti:sapphire has opened a way down towards the very short pulses (eg. reference 32). Table 5 gives the performances of the present BNL Nd:YAG laser together with the expected performances of the new Nd:YAG laser now under construction. 
Table 5: Parameters of the present and future BNL Nd:YAG lasers

\begin{tabular}{|c|c|c|}
\hline & Present & Future \\
\hline \multicolumn{3}{|l|}{ Single-Pulse Mode } \\
\hline \multicolumn{3}{|l|}{ Energy: } \\
\hline IR (total) & $20 \mathrm{~mJ}$ & $\geq 10 \mathrm{~mJ}$ \\
\hline IR into $2 \omega$ crystal & $8 \mathrm{~mJ}$ & $\leq 3 \mathrm{~mJ}$ \\
\hline Green & $1.6 \mathrm{~mJ}$ & $\leq 1 \mathrm{~mJ}$ \\
\hline UV & $100 \mu \mathrm{J}$ & $\leq 300 \mu \mathrm{J}$ \\
\hline Repetition rate & $1.5,3 \mathrm{~Hz}$ & $1.5,3 \mathrm{~Hz}$ \\
\hline \multicolumn{3}{|l|}{ Pulse duration (FWHM): } \\
\hline Oscillator IR & 100 ps & $17 \mathrm{ps}$ \\
\hline Amplified IR & $17-25 \mathrm{ps}$ & $24 \mathrm{ps}$ \\
\hline Green & $12-18 \mathrm{ps}$ & $19 \mathrm{ps}$ \\
\hline UV & $9-13 \mathrm{ps}$ & $15 \mathrm{ps}$ \\
\hline Beam diameter (FWHM) on cathode & $0.05-3 \mathrm{~mm}$ & $0.1,0.5,1 \mathrm{~mm}$ \\
\hline \multicolumn{3}{|l|}{ Shot to shot stability (on cathode, rms): } \\
\hline Timing & $\geq 3 \mathrm{ps}$ & $1 \mathrm{ps}$ \\
\hline Energy & $10 \%$ & $2 \%$ \\
\hline Pointing (1 mm beam diameter) & $\geq 100 \mu \mathrm{m}$ & $20 \mu \mathrm{m}$ \\
\hline Pointing ( $0.1 \mathrm{~mm}$ beam diameter) & $\geq 40 \mu \mathrm{m}$ & $20 \mu \mathrm{m}$ \\
\hline \multicolumn{3}{|l|}{ Pulse-Train Mode } \\
\hline Pulses in amplified train & $1-100$ & $1-200(100)$ \\
\hline Pulse spacing & $24.5 \mathrm{~ns}$ & $12.25(24.5) \mathrm{ns}$ \\
\hline IR energy per pulse & $1 \mathrm{~mJ}$ & $0.75(1.5) \mathrm{mJ}$ \\
\hline Pulse energy variation across train (rms) & $8 \%$ & $2 \%$ \\
\hline UV energy per pulse on cathode & & $40(80) \mu \mathrm{J}$ \\
\hline
\end{tabular}


The recent progress of diode pumping made possible the design of very compact, stable, reliable and relatively cheap Nd:YLF oscillators. More work has yet to be done to improve the performances of amplifiers and harmonic generation in terms of output energy, amplitude stability and beam quality.

Sophisticated feedback and feedforward loops are now being envisaged to improve the different types of stability. Temporal and spatial filters allow, in principle, the production of any longitudinal and transverse profile, at the expense of energy. These features are interesting for experimental tests of theoritical schemes developed to reduce the transverse emittance.

The R\&D on short pulse lasers relevant for photo-injector application is described in detail in references 42 and 43 .

\section{BEAM DYNAMICS}

RF gun beam dynamics was worked out in an analytical manner by K.J. Kim ${ }^{12}$. This simple model provides the gun designer with handy formulae for the different gun parameters, and are especially useful to understand the scaling of these parameters with such variables as the bunch length, the spot size on cathode, the peak accelerating field or the bunch charge. An improved model giving more accurate results, especially for the transverse bunch dimension was recently derived by L. Serafini ${ }^{18}$.

Most of the theoritical work done on RF gun beam dynamics concerns the possibility of obtaining smaller emittances, either by compensating the correlated emittance generated mainly in the first cell, or by removing the causes of extra emittance growth. Most of these techniques are reviewed in reference 44. Not all of them were experimentally proven or even tested. The most successfull one is the compensation of the correlated linear space charge induced emittance by the use of a magnetic focusing solenoid, due to B. Carlsten ${ }^{10}$. This technique experimentally proven at Los Alamos, allows a ten fold reduction of the emittance. Once thought unapplicable at high frequencies and high gradient, it will soon be implemented at $\mathrm{BNL}^{45}$.

Besides emittance compensation schemes, recent ideas being studied include travelling wave RF guns ${ }^{46}$ and asymmetric guns for linear colliders ${ }^{47}$.

\section{CONCLUSION}

Ten years after its invention, the photo-injector has reached the point where it is used daily at user facilities (e.g. BNL), and where it opened the way to new results such as UV FEL and the generation of $30 \mathrm{GHz}$ power. However, can it already be considered as a reliable technology?

Before answering this question, one can try to classify what are the main problems and difficulties encountered by the people who are operating photo-injectors. Table 6 shows the result of a survey the author made through the people in charge of the different photoinjectors that were presented above. It definitely shows that the laser is the most important issue. This is rather normal, since the lasers needed by photo-injector applications are quite 
specific and therefore were not ready in the catalogs waiting to be used. They need to undergo specific developments, and this of course takes time, effort and money.

Table 6: Survey on photo-injector current issues

\begin{tabular}{lc}
\hline \hline Laser stability (amplitude, phase, ...) & $38 \%$ \\
Photocathode (QE, lifetime, cost, RF contact, ...) & $15 \%$ \\
High gradient (dark current, ...) & $13 \%$ \\
Miscellaneous & $11 \%$ \\
Vacuum & $8 \%$ \\
Synchronization RF/laser & $5 \%$ \\
Cost & $5 \%$ \\
Instrumentation & $5 \%$ \\
\hline \hline
\end{tabular}

One of the questions of the above mentioned survey was: "Do you think photo-injector technology is mature enough to be used in a user facility for any kind of application? If not, when might it be? In spite of the issues currently faced by photo-injector builders and operators, the general response to this question was "yes". The details of the different answers are summarized in table 7 . Therefore, it seems that, provided that the remaining problems mainly concerning the laser stability and reliability are solved, the photo-injector is ready to replace the conventional DC gun + buncher injector for any kind of application.

Table 7: Answers to the question of maturity of the photo-injector technology

\begin{tabular}{l}
\hline \hline Yes \\
Yes, with a strong team of laser specialists \\
Yes, if enough money and manpower \\
Not perfect, but already used at some user facilities \\
Hope it will be soon \\
If compared to thermionic gun, no. OK in 96-97 \\
\hline \hline
\end{tabular}

\section{ACKNOWLEDGEMENTS}

The data and information for the different projects were kindly provided by I. Ben Zvi, S.C. Chen, J.P. Delahaye, A. Fischer, S. Joly, I. Madsen, R. Sheffield, P. Schoessow, M. Yoshioka. Discussions with P. Georges were appreciated when writing the laser section. This paper benefited from the discussions of the source working group during the Workshop. I would also like to thank P. Schoessow and A. Fry who kindly helped me to print my transparencies during the last two days before my talk, and T. Garvey who carefully red this manuscript. 


\section{REFERENCES}

1. Siemann R., "Overview of Linear Collider Designs", in Proceedings of the 1993 Particle Accelerator Conference, Washington, May 17-20, 1993.

2. Sheffield R., "Short Wavelength FELs", in Proceedings of the 1991 Particle Accelerator Conference, San Francisco, CA, May 6-9, 1991, pp. 1110-1114.

3. Schoessow P. et al., "The Argonne Wakefield Accelerator", in Proceedings of the European Particle Accelerator Conference, Nice, June 12-16, 1990, pp. 606-608.

4. Ben-Zvi I., "The BNL Accelerator Test Facility and Experimental Program", in AIP Proceedings 279, 1993, pp. 591-607.

5. Hübner K., "Two-beam Linear Colliders", in Proceedings of the International Conference on High Energy Accelerators, Hamburg, July 20-24, 1992.

6. Kung P. et al., "High-intensity Coherent FIR Radiation from Sub-picosecond Electron Bunches", SLAC-PUB-6426, 1994.

7. Amblard J., Belloni J., "Projet Elyse", unpublished report, Orsay University, June 1992.

8. Fraser J., Sheffield R., Nucl. Instr. \& Meth. A250 (1986), pp. 71-76.

9. Curtin M. et al., Nucl. Instr. \& Meth. A296 (1991), pp. 127-133.

10. Carlsten B., Nucl. Instr. \& Meth. A285 (1989), pp. 313-319.

11. Batchelor K. et al., "Operational Status of the Brookhaven National Laboratory Accelerator Test Facility", in Proceedings of the European Particle Accelerator Conference, Nice, June 12-16, 1990, pp. 541-543.

12. Kim K.J., Nucl. Instr. \& Meth. A275 (1989), pp. 201-218.

13. Joly S. et al., "Progress Report on the BRC Photo-Injector", in Proceedings of the European Particle Accelerator Conference, Nice, June 12-16, 1990, pp. 140-142.

14. Dowell D.H. et al., "First Operation of a High Duty Factor Photoinjector", in Proceedings of the Particle Accelerator Conference, Washington, May 17-20, 1993, pp. 2967-2969.

15. Michalke A. et al., "First Operation of the High Quantum Efficiency Photocathodes Inside Superconducting Cavities", in Proceedings of the European Particle Accelerator Conference, Berlin, March 24-28, 1992, pp. 1014-1016.

16. O’Shea P.G. et al., Physical Review Letters, Vol. 71, No 22, 1993.

17. Travier C. et al., "First Operation of a Femtosecond Laser Driven Photo-Injector", presented at the European Particle Accelerator Conference, London, June 27-July 1, 1994. 
18. Serafini L., "Analytical Description of Particle Motion in Radio-Frequency Photoinjectors", these Proceedings.

19. Westenskow G., Madey J., Laser and Particle Beams (1984), Vol. 2, Part 2, pp. 223-225.

20. Schultz D. et al., Nucl. Instr. \& Meth., A340 (1994), pp. 127-132.

21. Feldman D. et al., "Operation of the APEX Photoinjector Accelerator at $40 \mathrm{MeV}$ ", in Proceedings of the Linear Accelerator Conference, Ottawa, August 24-28, 1992.

22. Sheffield R. et al., Nucl. Instr. \& Meth. A341 (1994) pp. 371-374.

23. McDonald K.T., "Design of the Laser-Driven RF Electron Gun for the BNL Accelerator Test Facility", DOE/ER/3072-43, Princeton University, 1988.

24. Wang X.J. et al., "Measurements on Photoelectrons from a Magnesium Cathode in a Microwave Electron Gun", submitted to J. Appl. Phys., 1994.

25. Lehrman I. et al., Nucl. Instr. \& Meth. A318 (1992).

26. Baconnier Y. et al., "The CERN Linear Collider Test Facility", in Proceedings of the Linear Accelerator Conference, Ottawa, August 24-28, 1992, pp. 329-331.

27. Bossart R. et al., "Performances Obtained with the CERN Linear Collider Test Facility (CTF)", presented at the European Particle Accelerator Conference, London, June 27-July 1, 1994.

28. Delahaye J.P., private communication.

29. Yoshioka M. et al., "Development of an RF Gun that Uses a Laser-Triggered Photocathode for the Japan Linear Collider", in Proceedings of the International Conference on High Energy Accelerators, Hamburg, July 20-24, 1992.

30. Omori T. et al., "Development of Polarized Electron Source for Future Linear Colliders Using AlGaAs-GaAs Superlattice", in Proceedings of the International Conference on High Energy Accelerators, Hamburg, July 20-24, 1992.

31. Hartman S. et al., Nucl. Instr. \& Meth. A340 (1994), pp. 219-230.

32. Georges P. et al., "CANDELA Photo-Injector Drive Laser", in the Proceedings of the Particle Accelerator Conference, Washington, May 17-20, 1993, pp. 3053-3054.

33. Leblond B., Nucl. Instr. \& Meth. A317 (1992), pp. 365-372.

34. Gai W. et al., "The Argonne Wakefield Accelerator (AWA) Laser System and its Laser Pulse Shaper", in Proceedings of the Particle Accelerator Conference, Washington, May 17-20, 1993, pp. 3050-3052.

35. Power et al., "Witness Gun for the Argonne Wakefield Accelerator", in Proceedings of the Particle Accelerator Conference, Washington, May 17-20, 1993, pp. 3061-3062. 
36. Chen S.C. et al., "High Gradient Acceleration in a 17 GHz Photocathode RF Gun", in Proceedings of the Particle Accelerator Conference, Washington, May 17-20, 1993, pp. 2575-2577.

37. Proceedings of the Workshop on Photocathodes for Polarized Electron Sources for Accelerators, Stanford, September 8-10, 1993, SLAC-432.

38. Clendenin J. et al., Nucl. Instr. \& Meth. A340 (1994), pp. 133-138.

39. Logatchev P.V. et al., "Measurement of Time Response of Laser-Triggered GaAs Photocathode", presented at the European Particle Accelerator Conference, London, June 27-July 1, 1994.

40. Michelato P. et al., "Multialkali Thin Photocathodes for High Brightness Guns", presented at the European Particle Accelerator Conference, London, June 27-July 1, 1994.

41. Early J. et al., Nucl. Instr. \& Meth. A318 (1992), pp. 381-388.

42. Geissler K., Nucl. Instr. \& Meth. A340 (1994), pp. 52-73.

43. Proceedings of the Workshop on Lasers for RF Guns, Anaheim, May 14-15, 1994.

44. Serafini L., Nucl. Instr. \& Meth. A340 (1994), pp. 40-51.

45. Gallardo J.C., Kirk H., "An Injection Scheme for the Brookhaven ATF Utilizing SpaceCharge Emittance Growth Compensation", in Proceedings of the Particle Accelerator Conference, Washington, May 17-20, 1993.

46. Gao J., "Theoretical Investigation of Travelling Wave RF Gun", in Proceedings of the European Particle Accelerator Conference, Berlin, March 24-28, 1992, pp. 584-586.

47. Rosenzweig J. et al., "Design of a High Duty Cycle, Asymmetric Emittance RF Photocathode Injector for Linear Colider Applications", in Proceedings of the Particle Accelerator Conference, Washington, May 17-20, 1993, pp. 3021-3023. 